\title{
Caracterização de ninhos e tamanho de colônia de Acromyrmex rugosus (F. Smith) (Hymenoptera, Formicidae, Attini) em restingas de Ilhéus, $B A$, Brasil
}

\author{
Ilka Maria Fernandes Soares ${ }^{1,2}$, Terezinha Maria Castro Della Lucia ${ }^{2}$, Adriana Anadir dos Santos ${ }^{1}$, Ivan Cardoso \\ Nascimento $^{2} \&$ Jacques Hubert Charles Delabie 3
}

\begin{abstract}
${ }^{1}$ Departamento de Educação, Universidade do Estado da Bahia / Campus VIII, Rua do Gangorra, 503 - Alves de Souza, $48608-240$ Paulo Afonso-BA. ilka_soares@yahoo.com.br

${ }^{2}$ Departamento de Biologia Animal-Pós-Graduação em Entomologia, Universidade Federal de Viçosa, 36570-000 Viçosa-MG.

${ }^{3}$ U.P.A. Laboratório de Mirmecologia, Convênio UESC/CEPLAC, C.P. 07, 45600-000, Itabuna-BA e Universidade Estadual de Santa Cruz, Rodovia Ilhéus-Itabuna, Salobrinho.
\end{abstract}

\begin{abstract}
Characterization of nest and colony size of Acromyrmex rugosus (F. Smith) (Hymenoptera, Formicidae, Attini) in sandbanks at Ilhéus, BA, Brazil. Eight nests of Acromyrmex rugosus were excavated aiming to study their structure and to estimate their population. These nests were always found hidden by plants; presented a small heap of sandy soil and had 1 or 2 nest entrances; one of this had the shape of an inverted "U". Internally, the number of chambers was variable and the volume of fungus small $(\mathrm{X}=196 \mathrm{ml})$. The total nest population amounted, on the average, to 895 individuals.
\end{abstract}

KEYWORDS. Leaf-cutting ant; nest structure; population estimates.

RESUMO. Caracterização de ninhos e tamanho de colônia de Acromyrmex rugosus (F. Smith) (Hymenoptera, Formicidae, Attini) em restingas de Ilhéus, BA, Brasil. Oito ninhos de Acromyrmex rugosus foram escavados para estudar sua estrutura e estimar sua população. Esses ninhos eram sempre encontrados escondidos por plantas; apresentaram pequena quantidade de solo e tinham 1 ou 2 entradas; uma delas tinham a forma de um "U" invertido. Internamente, o número de câmaras foi variável e o volume de fungo foi pequeno $(\mathrm{X}=196 \mathrm{ml})$. O total da população do ninho foi aproximadamente, 895 indivíduos.

PALAVRAS-CHAVE. Estimativa populacional; estrutura de ninho; formiga cortadeira.

As formigas cortadeiras dos gêneros Atta e Acromyrmex possuem grande especialização na construção de ninhos. Estes se constituem da parte externa, caracterizada pela presença de terra solta e/ou, substrato vegetal seco e da parte interna, composta por câmaras (panelas) e túneis (canais) escavados no solo pelas operárias. Orifícios ligam a parte externa à interna. Existem câmaras para o cultivo do fungo simbionte, onde são alojados também os ovos, larvas, pupas e adultos, e câmaras para o lixo que contêm formigas mortas, fungo exaurido e vegetal seco. Em Acromyrmex spp., a área externa do ninho pode ser formada por terra solta ou não. Algumas espécies apresentam a área externa coberta por palha ou fragmentos vegetais. Os ninhos de Acromyrmex geralmente são inconspícuos, dificultando sua localização (Della Lucia \& Moreira 1993).

Acromyrmex rugosus rugosus tem larga distribuição geográfica, sendo encontrada, em áreas de cerrado e caatinga em quase todo o País, podendo afetar culturas como algodão, feijão e laranja; além de plantas silvestres como pau-ferro (Gonçalves 1961). Visando aumentar o conhecimento sobre $A$. rugosus rugosus, subespécie ainda pouco estudada (Fowler 1979), procurou-se caracterizar seus ninhos e estimar sua população. Este trabalho foi realizado em janeiro e fevereiro de 2004 nos litorais norte e sul da cidade de Ilhéus, BA. No litoral norte $\left(14^{\circ} 36^{\prime} 57^{\prime \prime} \mathrm{S} 39^{\circ} 03^{\prime} 40^{\prime \prime} \mathrm{W}\right)$ as escavações foram realizadas na praia de São Domingos e na Praia de Ponta da Tulha em três áreas da vila Barramares, sendo uma à beira-mar. No litoral sul, as escavações foram realizadas próximas à Praia do Cururupe. Na praia de São Domingos, bem como numa das áreas da Vila Barramares, os locais se caracterizavam por extensas plantações de coqueiros, sob as palhas dos quais se encontravam os formigueiros.

Oito ninhos foram escolhidos aleatoriamente nas áreas. Antes da escavação, realizada com cavador e enxada, fez-se a caracterização externa, determinou-se o número de aberturas (número de olheiros de ventilação e de trabalho), a altura e o comprimento da terra solta e caracterizou-se o olheiro principal. Internamente foram contadas o número de câmaras, a altura $\mathrm{e}$ o comprimento de cada uma e o tamanho total do formigueiro. Com o fungo, acondicionado em sacos plásticos, seu volume total foi estimado. Para cada colônia, com mais de $200 \mathrm{ml}$ de fungo, amostraram-se duas parcelas (de $100 \mathrm{ml}$ cada). Porções de fungo foram retiradas de diferentes lugares para a contagem 
Tabela I. Dimensões (cm) dos ninhos de Acromyrmex rugosus rugosus escavados no litoral norte e sul de Ilhéus-BA, jan-fev./ 2004. (D. P.) Desvio padrão; (*) Altura x Largura x Profundidade.

\begin{tabular}{|c|c|c|c|c|c|c|}
\hline Ninho & $\begin{array}{l}\text { Profundidade da } \\
\text { primeira câmara }\end{array}$ & $\begin{array}{c}\text { Número de } \\
\text { câmaras de fungo }\end{array}$ & $\begin{array}{c}\text { Tamanho da maior câmara } \\
\text { de fungo }(\mathrm{AxLxP})^{*}\end{array}$ & $\begin{array}{l}\text { Número de } \\
\text { olheiros }\end{array}$ & $\begin{array}{l}\text { Largura total } \\
\text { do ninho }\end{array}$ & $\begin{array}{r}\text { Profundidade } \\
\text { do ninho }\end{array}$ \\
\hline 1 & 20 & 2 & $13 \times 22 \times 16$ & 2 & 30 & 70 \\
\hline 2 & 18 & 2 & $10 \times 12 \times 5$ & 1 & 37 & 85 \\
\hline 3 & 10 & 3 & $12 \times 30 \times 20$ & 2 & 45 & 185 \\
\hline 4 & 9 & 1 & $21 \times 20 \times 24$ & 1 & 12 & 30 \\
\hline 5 & 13 & 1 & $10 \times 5 \times 4$ & 1 & 20 & 25 \\
\hline 6 & 12 & 1 & $10 \times 12 \times 10$ & 1 & 12 & 25 \\
\hline 7 & 29 & 6 & $12 \times 10 \times 10$ & 2 & 95 & 130 \\
\hline 8 & 20 & 3 & $20 \times 25 \times 20$ & 2 & 90 & 100 \\
\hline Média & 16,37 & 2,3 & $13,5 \times 17 \times 12,3$ & 1,5 & 42,62 & 81 \\
\hline D.P. & 6,69 & 1,68 & $6,6 \times 8,5 \times 8$ & 1,25 & 32,88 & 56 \\
\hline
\end{tabular}

de larvas, pupas, operárias e formas aladas. De posse desses dados, estimou-se a população total, extrapolando-se os valores obtidos nessas amostras de $100 \mathrm{ml}$ para todo o volume de fungo do ninho (Silva et al. 1981; Pereira \& Della Lucia 1998). Nos ninhos em que o volume de fungo foi igual ou inferior a $200 \mathrm{ml}$, todo o conteúdo foi usado na contagem da população à exceção do número de ovos, pois são muito pequenos e de difícil visualização, mesmo com auxílio de estereoscópio. Verificou-se também a distribuição das castas nas câmaras e os inquilinos associados aos ninhos.

Os ninhos escavados em solos arenosos apresentaram pequena quantidade de terra solta sobre as câmaras. $\mathrm{O}$ número de olheiros variou de 1a 2, e de modo geral, um era de trabalho e o outro, de ventilação, ou seja, não foram observados movimentos de entrada e saída de operárias. Os olheiros de trabalho têm uma forma de "U" invertido. Nos ninhos localizados sob as palhas de coqueiro os olheiros não têm formato definido, e a quantidade de terra solta é muito maior do que naqueles construídos fora delas, talvez porque esses ninhos estejam expostos indiretamente às chuvas. Em ninhos sob terra solta, as gramíneas e dicotiledôneas, que não são cortadas pelas formigas, ocultam os formigueiros. O número de olheiros e a camuflagem do formigueiro foram os mesmos observados por Gonçalves (1961) e Delabie et al (1997).

O número de câmaras nos formigueiros é variável (1-9), o que se deve, aparentemente, à idade da colônia e ao local de nidificação. Quando os ninhos são muito próximos dos coqueiros, esse número é menor, assim como sua profundidade (máximo $0,70 \mathrm{~m}$ ). Quando situados à beira-mar, onde há poucas raízes de plantas, a quantidade de câmaras e a profundidade podem ser de até $1,85 \mathrm{~m}$ (Tab. I).

O olheiro principal abre-se numa galeria curta $(6-29 \mathrm{~cm})$ que leva à primeira câmara com fungo, que são as maiores e geralmente sustentadas por raízes de coqueiros, gramíneas ou dicotiledôneas. A distribuição do fungo nas câmaras é irregular, ou seja, pode-se encontrá-lo tanto na parte inferior quanto na parte superior quando essas são sustentadas por raízes, à semelhança dos de Ac. balzani (Mendes et al. 1992). No entanto, ao contrário do que ocorre com esta espécie, em Ac. rugosus rugosus nem sempre as câmaras estão superpostas. Muitas vezes observa-se que algumas delas apresentam abertura lateral ou látero-posterior que leva a outras câmaras, normalmente, com fungo. Em geral, as câmaras têm formato ovóide ou riniforme; a altura varia de $6-21 \mathrm{~cm}$ e a largura de 5-30 cm (Tab. I), de modo similar aos ninhos de $A c$. balzani (Gonçalves 1961).

Nos formigueiros maiores, a rainha foi encontrada nas últimas câmaras de fungo, junto com uma grande quantidade de imaturos e nos pequenos, uma quantidade de ovos e larvas ou foi observada na primeira câmara de fungo onde a rainha

Tabela II. Composição da população dos ninhos de Acromyrmex rugosus rugosus escavados nos litorais norte e sul da cidade de Ilhéus-BA, janfev./ 2004

\begin{tabular}{|c|c|c|c|c|c|c|}
\hline \multirow[t]{2}{*}{ Ninho } & \multirow{2}{*}{$\begin{array}{l}\text { Volume de fungo } \\
(\mathrm{ml})\end{array}$} & \multicolumn{5}{|c|}{ Número médio de indivíduos } \\
\hline & & larvas & pupas & operárias adultas & alados & total \\
\hline 1 & 150 & 181 & 429 & 322 & - & 932 \\
\hline 2 & 150 & 209 & 374 & 422 & - & 1005 \\
\hline 3 & 180 & 83 & 17 & 383 & - & 483 \\
\hline 4 & 350 & 208 & 527 & 853 & - & 1588 \\
\hline 5 & 10 & 5 & 52 & 55 & - & 112 \\
\hline 6 & 80 & 36 & 50 & 107 & 02 & 195 \\
\hline 7 & 150 & 147 & 216 & 458 & 25 & 846 \\
\hline 8 & 500 & 365 & 112 & 1552 & 29 & 2058 \\
\hline Media \pm D.P. & $196,2 \pm 156,1$ & $154,2 \pm 114,8$ & $221,1 \pm 196,9$ & $519 \pm 483,1$ & $56 \pm 47,9$ & $895,3 \pm 667,7$ \\
\hline
\end{tabular}




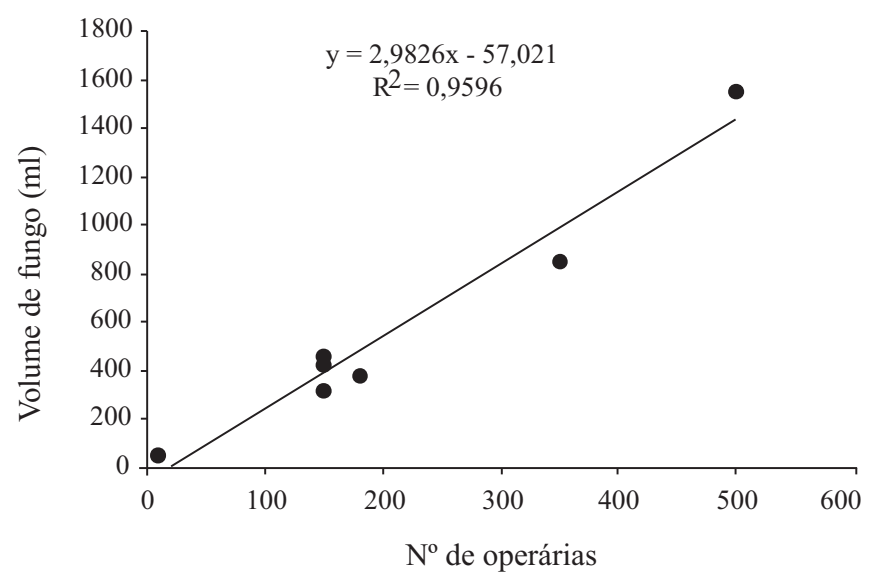

Fig.1. Influência do volume de fungo $(\mathrm{ml})$ sobre o número de operárias em Acromyrmex rugosus rugosus.

sempre se encontrava ou numa câmara pequena contígua a esta. Quando uma pequena quantidade de fungo foi encontrada na câmara que faz comunicação com a de fungo, encontravamse também as pupas.

$\mathrm{Na}$ câmara com lixo de dois ninhos foram encontrados vários Isopoda e Atarsocoris macroptera Becker, 1967 (Heteroptera, Cydnidae, Scaptocorinae), presos às raízes das plantas que dão sustentação às câmaras de fungo. A presença de Cydnidae, que comumente se alimenta de raízes, já foi relatada em ninhos de Atta, ocupando cavidades com detritos mais exauridos de A.texana (Moser 1963 e Froeschner 1960). A coexistência de inquilinos nos ninhos de formigas é muito comum em função das condições favoráveis de temperatura e umidade. Tais fatores do clima também podem contribuir para o desenvolvimento de determinados animais, como os Reptilia (Zolessi \& Gonzalez 1974; Oliveira \& Della Lucia 1993; Pilati \& Quiran 1996).

Nos ninhos escavados o volume de fungo variou entre 10$500 \mathrm{ml}$ (Tab. II) e o número de câmaras não esteve relacionado com esse volume. O formigueiro 8 apresentou maior quantidade de fungo $(500 \mathrm{ml})$ e apenas três câmaras, equivalente ao ninho 3, com apenas $180 \mathrm{ml}$. O formigueiro 7, cujo volume de fungo foi de $150 \mathrm{ml}$, continha 6 câmaras. No entanto, a quantidade de fungo está diretamente correlacionada ao número de operárias (Fig. 1), corroborando os dados obtidos por Silva et al. (1981), que demonstraram que a população de operárias cresce à medida que aumenta o volume do fungo, assim como com a população total. Esse mesmo resultado foi observado em Trachymyrmex fuscus, com volume de fungo de $350 \mathrm{ml}$ e 1035 indivíduos (Araújo et al. 2002), valor muito próximo ao observado em $A$. rugosus rugosus que, com a mesma quantidade de fungo, apresentou aproximadamente 1588 indivíduos. Em apenas três formigueiros foram encontradas formas aladas (Tab. II).

A população estimada variou de 112 a 2029 indivíduos e, com exceção do formigueiro 1 , o número de adultos foi sempre superior ao dos imaturos. O número de indivíduos por colônia foi pequeno (média de 519 operárias) (Tabela II) quando comparado com a de outras espécies do mesmo gênero, mas, foi semelhante à de operárias de $A$. balzani, estimada por Mendes et al. (1992) que registraram uma média de 417 indivíduos, enquanto em $A$. coronatus foi de 164.162 (Silva et al. 1988) e em Acromyrmex subterraneus subterraneus foi de 20.872 indivíduos (Pereira \& Della Lucia 1998).

Este trabalho permitiu concluir que em áreas de restinga os ninhos de Acromyrmex rugosus rugosus são difíceis de serem visualizados por estarem sob palhas de coqueiro, além da presença de plantas que ficam sobre a terra solta.

Agradecimentos. Ao CNPq e à Universidade do Estado da Bahia pela concessão de bolsas; ao Programa PRONEX/FAPESB ( $n^{\circ} 158 / 03$ ) pelo suporte financeiro, a Adson Oliveira pela ajuda nas escavações dos formigueiros, ao Prof. Paulo Sérgio Fiúza pela identificação de Heteroptera e à revisora de textos Rizele M.C. Reis, ambos da UFV.

\section{REFERÊNCIAS}

Araújo, M. S.; T. M. C. Della Lucia, \& A. J. May-Nunes. 2002. Caracterização de ninhos e atividade forrageadora de Trachymyrmex fuscus Emery (Hymenoptera, Formicidae) em plantio de eucalipto. Revista Brasileira de Zoologia 19: 419-427.

Delabie, J. H. C.; I. C. do Nascimento; E. Fonseca; R. B. Sgrilo; P. A. O. Soares; A. B. Casimiro \& M. Furst. 1997. Biogeografia das formigas cortadeiras (Hymenoptera; Formicidae; Myrmicinae; Attini), de importância econômica no leste da Bahia e nas regiões periferias dos estados vizinhos. Agrotrópica 9: 49-58.

Della Lucia, T. M. C. \& D. D. O. Moreira. 1993. Caracterização dos ninhos, p. 32-142 In: T. M. C. Della Lucia (ed.). As formigas cortadeiras. Viçosa, Folha de Viçosa, xiii+262 p.

Fowler, H. G. 1979. Environmental correlates of the foraging of Acromyrmex crassispinus. Ciência e Cultura 31: 879-882.

Froeschner, R. C. 1960. Cydnidae of the Western hemisphere. Proceedings of the U.S. Natural Museum 111: 337-680.

Gonçalves, C. R. 1961. O gênero Acromyrmex no Brasil (Hym. Formicidae). Studia Entomologica 4: 113-180.

Mendes, W. B. A.; J. A. H. Freire; M. C. Loureiro; S. B. Nogueira; E. F. Vilela \& T. M. C. Della Lucia. 1992. Aspectos ecológicos de Acromyrmex (Moellerius) balzani (EMERY, 1890) (Formicidae: Attini) no município de São Geraldo, Minas Gerais. Anais da Sociedade Entomológica do Brasil 21: 155-168.

Moser, J. C. 1963. Contents and structure of Atta texana nest in summer. Annals of the Entomological Society of America 56: 286291.

Oliveira, M. A. \& T. M. C. Della Lucia. 1993. Inquilinismo de Phylodryas olfersii (Reptilia, Squamata, Colubridae) em ninhos de Acromymrex subterraneus (Hymenopera, Formicidae, Attini). Revista Brasileira de Entomologia 37: 113-115.

Pereira, R. de C. \& T. M. C. Della Lucia. 1998. Estimativa populacional em ninhos de Acromyrmex subterraneus subterraneus Forel, 1893 (Hymenoptera: Formicidae). Revista Ceres 45: 573-578.

Pilati, A. \& E. Quiran. 1996. Patrones de cosecha de Acromyrmex lobicornis (Formicidae; Attini) en un pastizal del Parque Nacional Lihué Calel, La Pampa, Argentina. Ecología Austral 6: 123-126.

Silva, V. P.; L. C. Forti \& Z. G. Cardoso. 1981. Dinâmica populacional e caracterização dos ninhos de Acromyrmex coronatus (Fabricius, 1804) (Hymenoptera: Formicidae). Revista Brasileira de Entomologia 25: 87-93.

Zolessi, L. C. \& L. A. Gonzaléz. 1974. Nidificacion y mesoetologia de Acromyrmex en el Uruguay, II Acromyrmex lobicornis (Emery, 1887). (Hymenoptera: Formicidae) Revista de Biología del Uruguay 1: $37-57$. 